\title{
EFFECT OF SOME INSECTICIDES ON THE COTTON LEAF WORM Spodoptera littoralis (Boisd.) AND ITS ASSOCIATED PREDATOR (Coccinella septempunctata L ) ON TOMATO PLANTS \\ Ibrahim, Evleen G. \\ Plant Protection Research Institute, Agricultural Research Center, Sabahia, Alexandria
}

\begin{abstract}
In 2005 and 2006 tomato season, two experiments were conducted to study the effect of using five insecticides applied on cotton leaf-worm Spodoptera littoralis (Boisd) and its predator Coccinella septempunctata L.in tomato fields. Five pesticides Profenofos (Celeian 72\%Ec), Diflubenzaron (Diflurait 25\%WP), Methomyl (Mitholait 20\%SL), Chlorpyriphos-methyl (Birodan 50\%EC) and Fentrothyon (Adoya Fentro 50\% EC) were tested. Weekly counts of numbers second and fourth instar larvae on cotton leaf-worm and its predator were conducted in each plot. Results showed that during season 2005 the insecticide mitholait was the most potent chemical on the fourth instar larvae $(85.5 \% \mathrm{R})$, but it was the least on the second instar larvae $(60.7 \% \mathrm{R})$.

Diflurait gave highly reduction on the second instar larvae $(78.9 \% \mathrm{R})$. During season 2006, the results showed also, the insecticide mitholait was still the most effective one on the fourth instar larvae $(78.8 \% \mathrm{R})$ and the least effective one on the second instar larvae $(70.6 \% \mathrm{R})$, while the insecticide celeian gave the high effect on the second instar larvae $(80.2 \% \mathrm{R})$. During two seasons, the insecticide diflurait was the least effective one and did not detrimental effects on the predator $(70.9$ and $71.4 \%$ $\mathrm{R})$ respectively. This proves that combination of mitholait plus the diflurait may fit well into IPM programs.
\end{abstract}

Keywords: insecticides, cotton leaf-worm, predator.

\section{INTRODUCTION}

Cotton is still the most important agricultural crop in Egypt. It is always attacked by several pests, among them the cotton leaf-worm $S$. littoralis (Boisd.) it is a polyphagous insect, attacking a large number of host plants. The larval stage is the major enemy of several field crops, vegetables. Synthetic pesticides have been used for many years to control this pest. However, considerable problems arose from the continued application of these insecticides including the development of resistance by insects and the pollution of the environment.

Furthermore, several authors have discussed the effect of different types of insecticides, rates and methods of application on the number of predators in cotton fields in Egypt (Hafez, 1960 \& 1972, Ibrahim 1962, Hassanein \& Khalil 1968, Ibrahim \& Fayad 1980, Fayad \& Ibrahim 1981). Adult and larval stages of predators to Coccinellidae play an important role in regulating pest population (Ibrahim \& Fayad and Sarhan 1979, Ibrahim, 1983 and Kamal, 1951). 
Different species of coccinellid predators such as Coccinella septempunctata L. (F. Coccinellidae) which is considered as one of the biological control of the cotton leaf-worm (eggs and small larval) (Alfieri, 1976 and Wiesmann, 1955).

The present study throws light on the effect of some new insecticides of cotton leaf-worm and its predator.

\section{MATERIALS AND METHODS}

The present field experiments were carried out in khorishid region, Alexandria, during 2005 and 2006 seasons. Tomato plants (variety peto-68) were planted in June of both seasons, in an area of one feddan. All cultural practices and fertilizers were followed as commonly practiced. In all cases, the high volume spraying method by use of knapsack sprayers was used at the rate of $400 \mathrm{~L}$. of diluted spray/feddan. Also the experimental design was the randomized plot design, marking 4 replicates for each treatment as well as the untreated control. The results were based on counts on both leaf surfaces of living insects per sample of 10 leaves for each replicates, making one count before spraying and 3 counts at 3,5 and 7 day intervals after spraying on the second and fourth instar larvae of cotton leaf worm spodoptera $\mathrm{L}$. and its associated predator insect $C$. septempunctata $\mathrm{L}$ (adult). Percentages of reduction of infestation were calculated according to the equation of Henderson and Tilton (1955): (arcsin transformation was done) $\%$ Reduction of in festation $=100[1-(\mathrm{ta} / \mathrm{tb}) \times(\mathrm{cb} / \mathrm{ca})]$. where::

$\mathrm{Ta}=$ Post-treatment counts.

$\mathbf{T b}=$ Pre- treatment counts.

$\mathrm{Cb}=$ Untreated counts before treatment.

$\mathbf{C a}=$ Untreated counts after treatment.

L.S.D. statical analysis was employed to test the significance of differences between treatments (Snedecor, 1982).

Five insecticide were tested at the rates indicated in Table (1).

Table (1) treatment used of some insecticides on tomato plants.

\begin{tabular}{|l|c|c|c|}
\hline Trade marke & Common name & $\begin{array}{c}\text { Rate/faddan } \\
(\mathrm{L} .)\end{array}$ & Producing Company \\
\hline Celeian $72 \% \mathrm{EC}$ & Profenofos & $750 \mathrm{cc} / \mathrm{f}$ & $\mathrm{KZ}$ Company \\
\hline Diflurait $25 \% \mathrm{WP}$ & Diflubenzaron & $70 \mathrm{gm} / 100 \mathrm{~L}$ & Misr Agr. Developmen \\
\hline Mitholait $20 \% \mathrm{SL}$ & Methomyl & $1.25 \mathrm{~L} / \mathrm{f}$. & Misr Agr. Development \\
\hline Birodan $50 \% \mathrm{EC}$ & $\begin{array}{c}\text { Chlorpyriphos- } \\
\text { methyl }\end{array}$ & $1 \mathrm{lit} / \mathrm{f}$. & $\begin{array}{c}\text { The National company for } \\
\text { Agochemical production. }\end{array}$ \\
\hline Adoya fentro 50\% EC & Fentrothyon & $250 \mathrm{cc} / 100 \mathrm{~L}$ & Adoya Company. \\
\hline
\end{tabular}

\section{RESULTS AND DISCU SSION}

\section{A- Effect of insecticides on second instar larvae:}

The averages of percentages reduction of infestation during both seasons are given in Tables (2 \& 3). In the first season (2005), it could 
concluded that there were significant differences between all the tested insecticides and untreated control (L.S.D. $=0.504$ at $5 \%$ level of significance). These differences were evident in the highly percentage of reduction for treatment. Diflurait treatment gave the highest percentage of reduction (78.9\%), followed by Birodan (76.7\%), Celeian (71.8\%) and Adoyafentro (71.4\%) with no significant differences among Diflurait, Mitholait and Birodan compounds. On the other hand, the least percentage of reduction was obtained in Metholait treated plots. Results gave an average percentage of $(60.7 \%)$.

Table (2): Efficiency of some insecticides in controlling Spodoptera littoralis (Bosid) on tomato plants during season (2005).

\begin{tabular}{|c|c|c|c|c|c|c|c|c|c|c|}
\hline \multirow{3}{*}{ Treatments } & \multicolumn{5}{|c|}{$2^{\text {ed }}$ instar larvae } & \multicolumn{5}{|c|}{$4^{\text {th }}$ instar larvae } \\
\hline & \multirow{2}{*}{$\begin{array}{l}24 \mathrm{~h} \text {. pre- } \\
\text { treatment }\end{array}$} & \multicolumn{4}{|c|}{$\begin{array}{c}\text { Number of } 2^{\text {ed }} \text { larvae and } \\
\text { (\%R) at indicated days } \\
\text { post-treatment }\end{array}$} & \multirow{2}{*}{$\begin{array}{l}24 \mathrm{~h} \text { pre- } \\
\text { treatment }\end{array}$} & \multicolumn{4}{|c|}{$\begin{array}{l}\text { Number of } 4^{\text {th }} \text { larvae and } \\
(\% R) \text { at indicated days post } \\
\text { treatment }\end{array}$} \\
\hline & & \begin{tabular}{|c|}
3 \\
days
\end{tabular} & \begin{tabular}{|c}
5 \\
days
\end{tabular} & \begin{tabular}{|c|}
7 \\
days
\end{tabular} & \begin{tabular}{|c|} 
Average \\
$\%$ reduction
\end{tabular} & & \begin{tabular}{|c|}
3 \\
days
\end{tabular} & \begin{tabular}{|c}
5 \\
days
\end{tabular} & $\begin{array}{c}7 \\
\text { days }\end{array}$ & $\begin{array}{c}\text { Average } \\
\% \text { reduction }\end{array}$ \\
\hline$\frac{\text { Celeian }}{\frac{72 \%}{E C}}$ & 212 & $\begin{array}{c}21 \\
(91.1)\end{array}$ & $\begin{array}{c}90 \\
(64.8)\end{array}$ & $\begin{array}{c}124 \\
(59.6)\end{array}$ & (71.8) & 216 & $\begin{array}{c}26 \\
(90.1)\end{array}$ & $\begin{array}{c}68 \\
(65.0)\end{array}$ & $\mid \begin{array}{c}85 \\
(51.1)\end{array}$ & (68.7) \\
\hline $\begin{array}{l}\text { Diflurait } 25 \% \\
\text { WP }\end{array}$ & 410 & \begin{tabular}{|c|}
79 \\
$(82.7)$ \\
\end{tabular} & $\begin{array}{c}82 \\
(83.4)\end{array}$ & $\begin{array}{c}175 \\
(70.6)\end{array}$ & (78.9) & 384 & $\begin{array}{c}35 \\
(92.5)\end{array}$ & $\begin{array}{c}112 \\
(67.6)\end{array}$ & $\begin{array}{c}151 \\
(51.1)\end{array}$ & $(70.4)$ \\
\hline $\begin{array}{l}\text { Mitholait } \\
\text { 20\%SL }\end{array}$ & 357 & \begin{tabular}{|c|}
36 \\
$(90.9)$ \\
\end{tabular} & $\begin{array}{c}190 \\
(55.8) \\
\end{array}$ & \begin{tabular}{|c|}
334 \\
$(35.5)$ \\
\end{tabular} & $(60.7)$ & 418 & $\begin{array}{c}37 \\
(92.7) \\
\end{array}$ & $\begin{array}{c}41 \\
(89.1) \\
\end{array}$ & $\begin{array}{c}85 \\
(74.7) \\
\end{array}$ & (85.5) \\
\hline $\begin{array}{ll}\text { Birodan } & 50 \% \\
\text { EC } & \\
\end{array}$ & 255 & \begin{tabular}{|c|}
19 \\
$(95.3)$ \\
\end{tabular} & $\begin{array}{c}65 \\
(78.8) \\
\end{array}$ & $\begin{array}{c}240 \\
(56.1)\end{array}$ & $(76.7)$ & 432 & $\begin{array}{c}78 \\
(85.1)\end{array}$ & $\begin{array}{c}129 \\
(66.8) \\
\end{array}$ & $\begin{array}{c}138 \\
(60.3)\end{array}$ & $(70.7)$ \\
\hline $\begin{array}{l}\text { A } \quad \text { doyafentro } \\
50 \% \text { EC }\end{array}$ & 309 & $\begin{array}{c}26 \\
(92.4) \\
\end{array}$ & $\begin{array}{c}141 \\
(62.1) \\
\end{array}$ & $\begin{array}{c}180 \\
(59.8) \\
\end{array}$ & (71.4) & 283 & $\begin{array}{c}84 \\
(75.5) \\
\end{array}$ & $\begin{array}{c}92 \\
(63.9)\end{array}$ & $\begin{array}{c}101 \\
(55.6)\end{array}$ & $(65.0)$ \\
\hline Untreated & 200 & $\begin{array}{l}222 \\
(\ldots)\end{array}$ & $\begin{array}{l}240 \\
(\ldots)\end{array}$ & $\begin{array}{l}290 \\
(\ldots)\end{array}$ & $(\ldots)$ & 520 & $\begin{array}{l}629 \\
(\ldots)\end{array}$ & $\begin{array}{l}468 \\
(\ldots)\end{array}$ & $\begin{array}{l}418 \\
(\ldots)\end{array}$ & $(\ldots)$ \\
\hline
\end{tabular}

L.S.D. (Pest) 0.05 level $=0.504$

L.S.D. (Time) 0.05 level $=0.619$

Total number of $2^{\text {ed }} \& 4^{\text {th }}$ instar larvae on 40 leaves

Table (3): Efficiency of some insecticides in controlling Spodoptera littoralis (Bosid) on tomato plants during season (2006).

\begin{tabular}{|c|c|c|c|c|c|c|c|c|c|c|}
\hline \multirow{3}{*}{ Treatments } & \multicolumn{5}{|c|}{$2^{\text {ed }}$ instar larvae } & \multicolumn{5}{|c|}{$4^{\text {th }}$ instar larvae } \\
\hline & \multirow{2}{*}{$\begin{array}{l}24 \text { h. pre- } \\
\text { treatment }\end{array}$} & \multicolumn{4}{|c|}{$\begin{array}{c}\text { Number of } 2^{\text {ed }} \text { larvae and } \\
(\% R) \text { at indicated days } \\
\text { post-treatment }\end{array}$} & \multirow{2}{*}{$\begin{array}{c}24 \mathrm{~h} \text { pre- } \\
\text { treatment }\end{array}$} & \multicolumn{4}{|c|}{$\begin{array}{c}\text { Number of } 4^{\text {th }} \text { larvae and } \\
(\% R) \text { at indicated days post } \\
\text { treatment }\end{array}$} \\
\hline & & \begin{tabular}{|c|}
3 \\
days
\end{tabular} & $\begin{array}{c}5 \\
\text { days }\end{array}$ & \begin{tabular}{|c|}
7 \\
days
\end{tabular} & \begin{tabular}{|c|} 
Average \\
\%reduction
\end{tabular} & & $\begin{array}{c}3 \\
\text { days }\end{array}$ & \begin{tabular}{|c|}
5 \\
days
\end{tabular} & \begin{tabular}{|c|}
7 \\
days
\end{tabular} & \begin{tabular}{|c|} 
Average \\
$\%$ reduction
\end{tabular} \\
\hline$\frac{\text { Celeian }}{\frac{72 \%}{E C}}$ & 178 & $\begin{array}{c}12 \\
(92.8)\end{array}$ & $\begin{array}{c}28 \\
(85.5)\end{array}$ & $\begin{array}{c}87 \\
(62.4)\end{array}$ & $(80.2)$ & 109 & $\begin{array}{c}9 \\
(89.8))\end{array}$ & $\begin{array}{c}35 \\
(57.7)\end{array}$ & $\begin{array}{c}28 \\
(58.3)\end{array}$ & $(68.6)$ \\
\hline $\begin{array}{l}\text { Diflurait } 25 \% \\
\text { WP }\end{array}$ & 218 & $\begin{array}{c}20 \\
(90.2)\end{array}$ & $\begin{array}{c}73 \\
(69.1)\end{array}$ & \begin{tabular}{|c|}
127 \\
$(55.2)$ \\
\end{tabular} & $(71.5)$ & 79 & $\begin{array}{c}6 \\
(90.6)\end{array}$ & $\begin{array}{c}17 \\
(71.7)\end{array}$ & $\begin{array}{c}23 \\
(52.7))\end{array}$ & (71.7) \\
\hline $\begin{array}{l}\text { Mitholait } \\
20 \% \text { SL }\end{array}$ & 232 & $\begin{array}{c}22 \\
(89.9)\end{array}$ & $\begin{array}{c}78 \\
(68.9)\end{array}$ & $\begin{array}{c}142 \\
(52.9)\end{array}$ & $(70.6)$ & 86 & $\begin{array}{c}5 \\
(92.8)\end{array}$ & $\begin{array}{c}11 \\
(83.2)\end{array}$ & $\begin{array}{c}21 \\
(60.3)\end{array}$ & (78.8) \\
\hline $\begin{array}{l}\text { Birodan } 50 \% \\
\text { EC }\end{array}$ & 185 & $\begin{array}{c}15 \\
(91.4)\end{array}$ & $\begin{array}{c}53 \\
(73.5)\end{array}$ & $\begin{array}{c}112 \\
(53.3)\end{array}$ & (72.7) & 103 & $\begin{array}{c}6 \\
(92.8)\end{array}$ & $\begin{array}{c}25 \\
(68.1)\end{array}$ & $\begin{array}{c}30 \\
(52.7)\end{array}$ & $(71.2)$ \\
\hline$\frac{\text { A } \quad \text { doyafentro }}{50 \% \text { EC }}$ & 238 & $\begin{array}{c}26 \\
(88.3)\end{array}$ & $\begin{array}{c}56 \\
(78.3)\end{array}$ & $\begin{array}{c}131 \\
(57.6)\end{array}$ & $(74.7)$ & 63 & $\begin{array}{c}6 \\
(88.2)\end{array}$ & $\begin{array}{c}20 \\
(58.2)\end{array}$ & $\begin{array}{c}18 \\
(53.6)\end{array}$ & $(66.7)$ \\
\hline Untreated & 244 & $\begin{array}{l}229 \\
(\ldots)\end{array}$ & $\begin{array}{l}264 \\
(\ldots)\end{array}$ & $\begin{array}{l}317 \\
(\ldots)\end{array}$ & $(\ldots)$ & 104 & $\begin{array}{c}84 \\
(\ldots)\end{array}$ & $\begin{array}{c}79 \\
(\ldots)\end{array}$ & $\begin{array}{c}64 \\
(\ldots)\end{array}$ & $(\ldots)$ \\
\hline
\end{tabular}

L.S.D. (Pest) 0.05 level $=3.222$

L.S.D. $($ Time) 0.05 level $=4.652$ 
Total number of $2^{\text {ed }} \& 4^{\text {th }}$ instar larvae on $\mathbf{4 0}$ leaves

Also, there are significant differences among three time (L.S.D. $=0.619$ at 5\% level). In 2006 season, statistical analysis also indicated significant different between the tested chemicals and untreated control. Celeian treatment gave the highest percentage of reduction were $(80.2 \%)$.

Moderate percentage reduction were obtained by Adoyafentra $(74.7 \%)$, Birodan (72.7\%), Diflurait (71.5\%) and Mitholait (70.6\%) without significant differences between Celeian and Birodan compounds

(L.S.D. $=3.222$ at $5 \%$ level of significance). Also, there are significant differences among three time

(L.S.D. $=4.652$ at $5 \%$ level of significance).

\section{B- Effect of insecticides on fourth instar larvae:}

The results of these tests are given in Tables $(2 \& 3)$ During the first season (2005), statistical analysis indicated highly significant differences between the treatment and untreated ((L.S.D. $=0.504$ at $5 \%$ level of significance). (85.5\%).

Mitholait showed the highest percentages of reduction which reached

Birodan and Diflurait came next and showed almost similar percentages reduction $(70.7 \%, 70.4 \%$, respectively). Celeian showed a moderate effect (68.7\% reduction), and Adoyfentro showed the lowest effect (65.0\% reduction).

In the experiment of 2006, analysis of variance showed high significant differences among insecticides ((L.S.D. $=3.222$ at $5 \%$ level of significance).

Mitholait gave the highest mean percentage of reduction (78.8\%) compared with $66.7 \%$ for Adoyafentro. Diflurait and Birodan came next gave almost similar percentages reduction $(71.7 \%$ and $71.2 \%$, respectively). These results with those of the first season (2005).

Mitholait seemed to be the most promising insecticide for the control of cotton leaf-worm followed by Birodan and Diflourait, while Celeian and Adayfentro were less effective.

\section{C- Effect of insecticides on insect predator:}

The results are given in Table (4). Analysis of variance during first season (2005) revealed not significant differences among the treatments on C. septempunctata L

(L.S.D. $=15.045$ at $5 \%$ level). Plots treated with Birodan gave the best control (90.3\% reduction). Celeian (85.4\%) and Adoyafentro $(78,1 \%)$ came next in that respect. Moderat percentage reduction were obtain by Mitholait (75.7\%), while Diflurait gave the least control (70.9\% reduction).

In the experiment of 2006 season, statistical analysis indicated not significant differences among the insecticidal treatments ((L.S.D. $=10.798$ at $5 \%$ level of significance).

Birodan was the most effective (90.9\% reduction), compared with $87.9 \%$ for Celeian and $80.7 \%$ for Adoyafentro and $84.6 \%$ for Mitholail. On the other hand, Diflurait showed the least percentages of reduction (71.4\%). 
Table (4): Efficiency of some insecticides on associated predator insect with Spodoptera Littoralis on tomato plants.

\begin{tabular}{|c|c|c|c|c|c|c|c|c|c|c|}
\hline \multirow{3}{*}{ Treatments } & \multicolumn{5}{|c|}{2005 season } & \multicolumn{5}{|c|}{2006 season } \\
\hline & \multirow{2}{*}{ 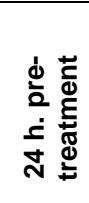 } & \multicolumn{4}{|c|}{$\begin{array}{c}{ }^{* \star} \text { Number of predator and } \\
(\% \mathrm{R}) \text { at indicated days post- } \\
\text { treatment }\end{array}$} & \multirow{2}{*}{ 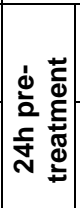 } & \multicolumn{4}{|c|}{$\begin{array}{l}\text { ** Number of predator }(\% R) \text { at } \\
\text { indicated days post treatment }\end{array}$} \\
\hline & & $\begin{array}{c}3 \\
\text { days }\end{array}$ & $\begin{array}{c}5 \\
\text { days }\end{array}$ & $\begin{array}{c}7 \\
\text { days }\end{array}$ & $\begin{array}{l}\text { Average } \\
\% \text { reduction }\end{array}$ & & $\begin{array}{c}3 \\
\text { days }\end{array}$ & $\begin{array}{c}5 \\
\text { days }\end{array}$ & $\begin{array}{c}7 \\
\text { days }\end{array}$ & $\begin{array}{l}\text { Average } \\
\text { \%reduction }\end{array}$ \\
\hline Celeian $72 \%$ EC & 6 & \begin{tabular}{|c|}
2 \\
$(70.8)$ \\
\end{tabular} & $\begin{array}{c}1 \\
(85.4) \\
\end{array}$ & $0(100)$ & $(85.4)$ & 4 & $1(78.6)$ & $1(85.0)$ & $\begin{array}{c}0 \\
(100)\end{array}$ & $(87.9)$ \\
\hline Diflurait $25 \%$ WP & 2 & $\begin{array}{c}1 \\
(56.3))\end{array}$ & $\begin{array}{c}1 \\
(56.3)\end{array}$ & $0(100)$ & $(70.9)$ & 3 & $3(14.3)$ & $0(100)$ & $\begin{array}{c}0 \\
(100)\end{array}$ & $(71.4)$ \\
\hline Mitholait $20 \%$ SL & 6 & $\begin{array}{c}3 \\
(56.3)\end{array}$ & $\begin{array}{c}2 \\
(70.8)\end{array}$ & $0(100)$ & $(75.7)$ & 5 & $2(65.7)$ & $1(88.0)$ & $\begin{array}{c}0 \\
(100)\end{array}$ & $(84.6)$ \\
\hline Birodan $50 \%$ EC & 3 & $\begin{array}{c}1 \\
(70.8)\end{array}$ & $\begin{array}{c}0 \\
(100)\end{array}$ & $0(100)$ & $(90.3)$ & 7 & $2(75.5)$ & $2(97.1)$ & $\begin{array}{c}0 \\
(100)\end{array}$ & $(90.9)$ \\
\hline $\begin{array}{l}A \text { day afentro } \\
50 \% \text { EC }\end{array}$ & 4 & $\begin{array}{c}2 \\
(56.3)\end{array}$ & $\begin{array}{c}1 \\
(78.1)\end{array}$ & $0(100)$ & $(78.1)$ & 4 & $2(57.1)$ & $1(85.0)$ & $\begin{array}{c}0 \\
(100)\end{array}$ & $(80.7)$ \\
\hline Untreated & 7 & $8(\ldots)$ & $8(\ldots)$ & $10(\ldots)$ & $(\ldots)$ & \begin{tabular}{|l|}
6 \\
\end{tabular} & $7(\ldots)$ & $10(\ldots)$ & $10(\ldots)$ & $(\ldots)$ \\
\hline $\begin{array}{l}\text { L.S.D. (Pest) } \\
\text { L.S.D. (Time) } \\
{ }^{*} \quad \text { Predator }= \\
{ }^{\star \star} \text { Total no. of } \\
\text { Coccinella Se }\end{array}$ & $\begin{array}{l}0.05 \text { lev } \\
.05 \text { leve } \\
\text { adult ar }\end{array}$ & $\begin{array}{l}\text { el }=15 \\
l=19 . \\
\text { ad larv } \\
r \text { on } 40 \\
\text { nctatal }\end{array}$ & $\begin{array}{l}5.045 \\
.566 \\
\text { ae } \\
0 \text { leave }\end{array}$ & 1 & 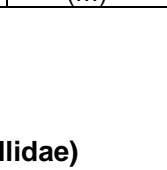 & $\begin{array}{l}\text { L.S.D } \\
\text { L.S.D }\end{array}$ & $\begin{array}{l}\text { (Pest) } \\
\text { (Time }\end{array}$ & $0.05 \mathrm{I}$ & $\begin{array}{l}\text { evel = } \\
\text { evel = } 2\end{array}$ & $\begin{array}{l}10.798 \\
21.702\end{array}$ \\
\hline
\end{tabular}

\section{Conclusion:}

In general, the results of the present study reveal that Mitholait was the most effective against fourth larvae (4th larvae) cotton leaf-worm, while it gave

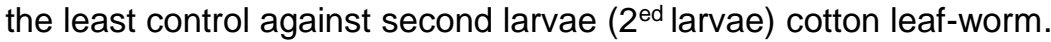

Birodan gave highly reduction against predators Coccinella which conceder as natural enemies in regulating some insect pest and for the biological control of cotton leaf-worm, Kamal 1951 and Ibrahim 1983). These insecticides could be useful only in cases of highly or moderate infestation.

\section{Acknowledgement}

I wish to express my deepest gratitude's to Prof. Dr. Omar Kolaib, Head of Economic Entomology and Agricultural Zoology Department, Faculty of Agriculture, Monoufia University, for reviewing this work. Many thanks go also to Prof. Dr. Ahamed Ebeda, Sabahia Research Station, Alex., for his valuable assistance in the statistical analysis. 


\section{REFERENCES}

Alfieri, A. (1976): The Coleoptera of Egypt. by Societe Entomologique D’Egypte: 5:pp.361, Cairo, Egypt.

Fayad, Y. H. and A. A. Ibrahim (1981): Effect of some new insecticides of cotton leaf-worm on the number of predators in cotton fields. (Proc; $1^{\text {st }}$. Conf. PL. Prot. Res. Ins.2:337 - 348).

Hafez, Mostafa (1960): The effect of some new insecticides on predators on the cotton leaf-worm in cotton fields. (Agric. Res. Rev. Cairo, 38: 4779).

Hafez, Mostafa (1972): Methods of Integrated control in cotton statement from Arab Republic of Egypt.(International cotton Advisory Committee, Oct. 1972, Managua, Nicaragua: 30-58).

Hassanein, M.H and F. M. Khalil (1968): Effect of insecticides on. Predators of the leaf-worm (Bull. Soc. Ent. Egypt, Econ., 2: 247-264).

Henderson, C. F. and E.W. Tilton (1955): Tests with acaricides against the brown wheat mite.J. Econ. Entomol., 48: 157-161.

Ibrahim, M. M. (1962): An indication of the effect of the widespread use of pesticides on the population of some predators in cotton fields. (Bull. Soc. Ent. Egypt, 46: 317-323).

Ibrahim, A.A. Y.H. Fayad and A.A. Sarhan (1979): Abundance of predators in three different cotton fields at Minya Governorate in 1976 cotton season. (Agric. Res. Rev. (1), 57: 65-70.

Ibrahim, A. A. and Y. H. Fayad (1980): Effect of granule insecticides on number of predators in cotton fields in Egypt. (J. Agric. Res. Tanta Univ. 6, (1): 242-248).

Ibrahim, L.I. (1983): Efficiency of some natural enemies in regulating some insect pest population. Ph. D. thesis, Faculty of Agric. Al-Azhar Univ., Cairo, Egypt.

Kamal, M.Bey (1951): The biological control of the cotton leaf-worm (Prodenia litura F.) in Egypt. Bull. Soc. Foud Ent., 35: 221-271).

Snedecor, G. W. and W. G. Cochran (1982): Statistical methods, $\left(7^{\text {th }}\right.$ ed. lowa state University press, Ames).

Wiesmann, R. (1955): Untersachungen an den predatoren der Baumwollschadinsekten in Jahre 1951-52 (Investigations on the predators of the insect pests of cotton in Egypt in the year 1951-52) Aeta. Trop. 12: 222-239. 
تأثير بعض مبيدات الآفات علي دودة ورق القطن والمفترس المرتبط بها (أبو العيد سبع نقط) علي نباتات الطماطم الطبر

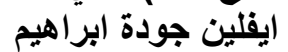
معهد بحوث وقاية النباتات ـ مركز البحوث الزراعيةــ الصبحية_الإسكندرية

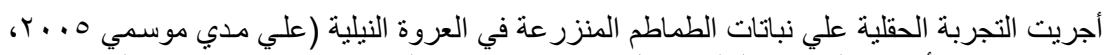

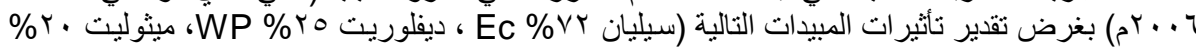

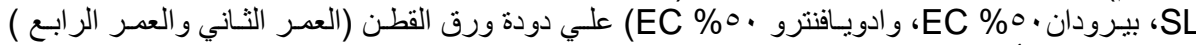

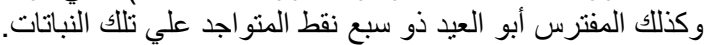

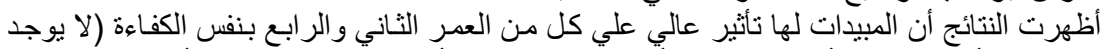

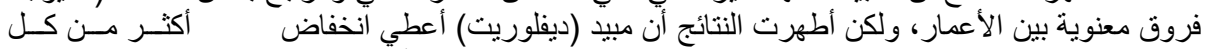

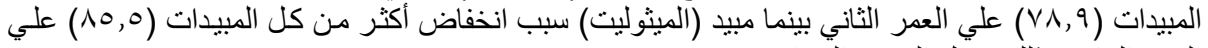

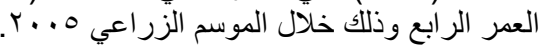

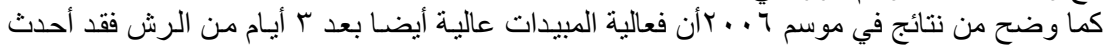

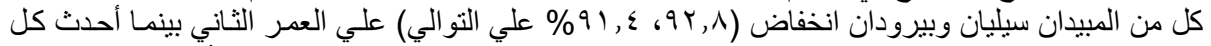

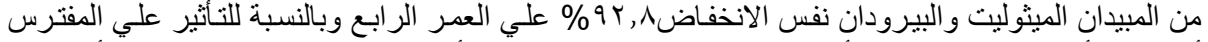

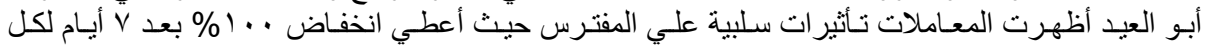

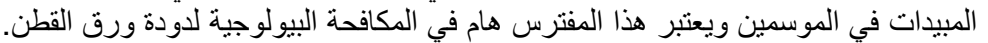

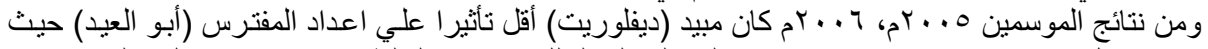

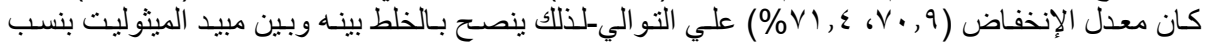
معينة و هذا قد يحقق نجاح في برنامج الإنغاض المكافحة المتكاملة. 